\title{
Solar Drivers of 11-yr and Long-Term Cosmic Ray Modulation
}

\author{
E.W. Cliver $\cdot$ I.G. Richardson $\cdot$ A.G. Ling
}

Received: 4 October $2010 /$ Accepted: 14 January 2011

(C) US Government 2011

\begin{abstract}
In the current paradigm for the modulation of galactic cosmic rays (GCRs), diffusion is taken to be the dominant process during solar maxima while drift dominates at minima. Observations during the recent solar minimum challenge the pre-eminence of drift at such times. In 2009, the $\sim 2$ GV GCR intensity measured by the Newark neutron monitor increased by $\sim 5 \%$ relative to its maximum value two cycles earlier even though the average tilt angle in 2009 was slightly larger than that in $1986\left(\sim 20^{\circ}\right.$ vs. $\left.\sim 14^{\circ}\right)$, while solar wind $B$ was significantly lower $(\sim 3.9 \mathrm{nT}$ vs. $\sim 5.4 \mathrm{nT})$. A decomposition of the solar wind into high-speed streams, slow solar wind, and coronal mass ejections (CMEs; including postshock flows) reveals that the Sun transmits its message of changing magnetic field (diffusion coefficient) to the heliosphere primarily through CMEs at solar maximum and high-speed streams at solar minimum. Long-term reconstructions of solar wind $B$ are in general agreement for the $\sim 1900$-present interval and can be used to reliably estimate GCR intensity over this period. For earlier epochs, however, a recent ${ }^{10} \mathrm{Be}$-based reconstruction covering the past $\sim 10^{4}$ years shows nine abrupt and relatively short-lived drops of $B$ to $\lesssim 0 \mathrm{nT}$, with the first of these corresponding to the Spörer minimum. Such dips are at variance with the recent suggestion that $B$ has a minimum or floor value of $\sim 2.8 \mathrm{nT}$. A floor in solar wind $B$ implies a ceiling in the GCR intensity (a permanent modulation of the local interstellar spectrum) at a given energy/rigidity. The $30-40 \%$ increase in the intensity of $2.5 \mathrm{GV}$ electrons observed by Ulysses during the recent solar minimum raises an interesting paradox that will need to be resolved.
\end{abstract}

E.W. Cliver $(\bowtie)$

Space Vehicles Directorate, Air Force Research Laboratory, Hanscom AFB, MA, USA

e-mail: afrl.rvba@hanscomat.mil

I.G. Richardson

Code 661, NASA/Goddard Space Flight Center, Greenbelt, MD, USA

I.G. Richardson

CRESST/Department of Astronomy, University of Maryland, College Park, MD, USA

A.G. Ling

Atmospheric and Environmental Research, Inc., Lexington, MA, USA

Published online: 24 February 2011

Springer 
Keywords Sun $\cdot$ Solar Cycle $\cdot$ Solar Wind $\cdot$ Coronal Mass Ejections $\cdot$ High-speed Streams · Galactic Cosmic Rays · Cosmic Ray Modulation

\section{Introduction}

Forbush (1954, 1958) discovered the 11-yr galactic-cosmic-ray (GCR) modulation cycle and noted its anti-correlation with the sunspot cycle. Parker (1965), Gleeson and Axford (1967), Jokipii and Parker (1970), and others [see Moraal 1976, 2011 (this issue), Potgieter 1998, and Heber and Potgieter 2006 for reviews] developed the particle transport equation which has terms governing GCR diffusion, convection, curvature/gradient drift, and adiabatic deceleration in the heliosphere. During the past three decades, modulation research has been primarily focused on understanding and specifying the roles of two of these termsdiffusion and curvature/gradient drift:

(1) In the diffusion-centered view, modulation results from the outward propagation, at the solar wind speed, of large steplike decreases in the GCR intensity (McDonald et al. 1981; cf., Morrison 1956, and Lockwood 1960). The disturbances responsible for these decreases came to be called "merged interaction regions" (MIRs) or "global merged interaction regions" (GMIRs; Burlaga et al. 1984, 1985, 1993; McDonald et al. 1993).

(2) Alternatively, the approach championed by Jokipii and colleagues (Jokipii et al. 1977; Jokipii and Thomas 1981; Kóta and Jokipii 1983) stresses the importance of curvature and gradient drifts of GCRs in the large-scale field of the heliosphere. In an early success of the drift picture, Jokipii and Thomas (1981) predicted the peaked cosmic ray intensity maximum in 1987 during the solar minimum between cycles 21 and 22 (Smith 1990).

Today, these two approaches—one emphasizing propagating diffusive barriers (e.g., Perko and Fisk 1983; Burlaga et al. 1985) and the other focusing on particle drift effects as parameterized by the solar "tilt" angle (e.g., Jokipii and Thomas 1981; Smith and Thomas 1986; Le Roux and Potgieter 1992a, 1992b)-have been combined to form the current paradigm for GCR modulation (e.g., Jokipii and Wibberenz 1998; Potgieter 2008, 2010). In this picture, drift effects are dominant on the decline of the solar cycle and at solar minimum while diffusion is dominant at solar maximum.

The current picture of modulation at solar maximum was challenged by Cane et al. (1999) and Wibberenz et al. (2002) who argued that since steplike decreases in the GCR intensity were first observed at Earth and then later in the outer heliosphere, it was incongruous to attribute them to MIRs or GMIRs formed by the merger/coalescence of coronal mass ejections (CMEs), shocks, and high-speed streams between 10-15 AU. Instead, they attributed modulation to global changes in the Sun's magnetic field. Subsequently, Sheeley et al. (2001), Cliver and Ling (200lb), and Wang et al. (2006) focused on changes of the Sun's low-latitude or non-axisymmetric open flux, which are more closely related to the sunspot number and solar wind $B$, than is the total open flux. More recently, the behavior of the Sun and the related cosmic ray response during the extended solar minimum period following cycle 23 have raised questions about the dominance of drift at this phase of the cycle.

In this study, we reinvestigate the solar and solar wind drivers of GCR modulation, both for 11-yr and longer-term modulation. Our focus in this paper is on the modulation of highrigidity $(\sim 2 \mathrm{GV})$ positively charged particles at $1 \mathrm{AU}$. Our analysis is presented in Sect. 2 and the results are summarized and discussed in Sect. 3. 


\section{Analysis}

\subsection{Diffusion vs. Drift}

Figure 1 contains timelines of solar wind $B$, the cosmic ray intensity (inverted) measured by the Newark neutron monitor (cutoff rigidity of $\sim 2 \mathrm{GV}$ ), and the sunspot number for solar cycles 20-23. In drift formalism, the intervals between maxima of the cycles are designated as $A$ positive or $A$ negative where $A$ positive (negative) cycles correspond to those for which the Sun's dipole magnetic field points outward (inward) in the northern hemisphere. During epochs when $A>0$, positively charged GCRs approach the inner heliosphere from over the poles (Jokipii and Kopriva 1979). In epochs when $A<0$, the preferred direction of approach of positively charged GCRs is inward along the heliospheric current sheet (HCS) that separates oppositely-directed fields in the northern and southern hemispheres of the heliosphere. The average excursion of the HCS from the heliographic equator is referred to as the tilt angle $(\alpha)$ of the heliospheric current sheet (available at http://wso.stanford.edu/). In practice $\alpha$ is defined as the average of the maximum angular excursions of the coronal neutral line (the base of the HCS) north and south of the Sun's equator during a 27-day solar rotation. The tilt angle is widely used to characterize drift effects on cosmic ray intensity, and we shall do so in this study. It should be kept in mind, however, that tilt angles provide only a crude approximation of the magnetic architecture of the heliosphere and that more sophisticated parameters, such as the total length of the wavy current sheet between Earth and the outer modulation boundary (e.g., Le Roux and Potgieter 1990) are needed.

Figure 2 contains scatter plots of 27-day averages of the GCR intensity measured at Newark vs. the tilt angle for $A$ positive (a) and $A$ negative (b) epochs, from 1976 to 2009 , the start of cycle 21 to the end of cycle 23. In each plot, blue filled points indicate data

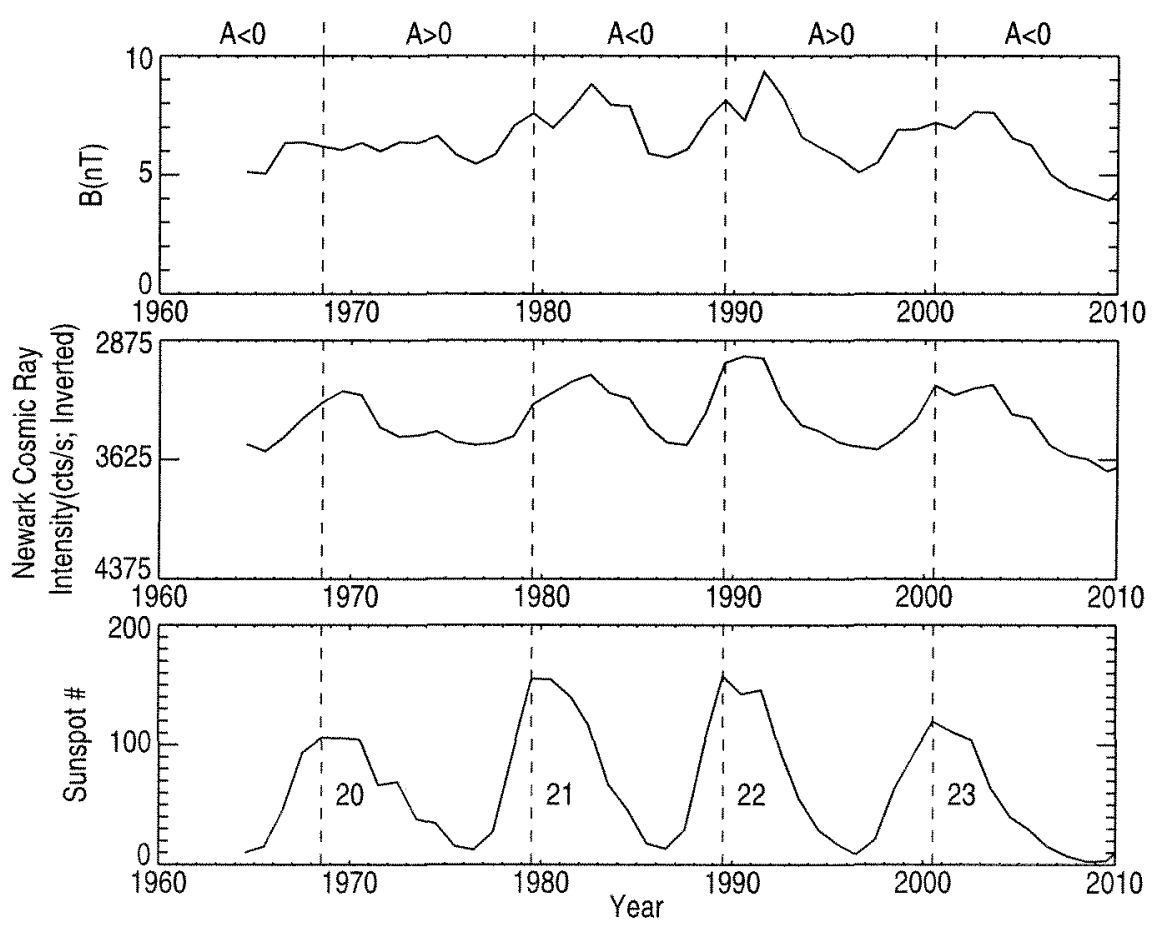

Fig. 1 Solar wind $B$, Newark cosmic ray intensity (inverted), and sunspot number, 1964-2009. The Sun's $A$ positive and $A$ negative magnetic epochs are indicated 


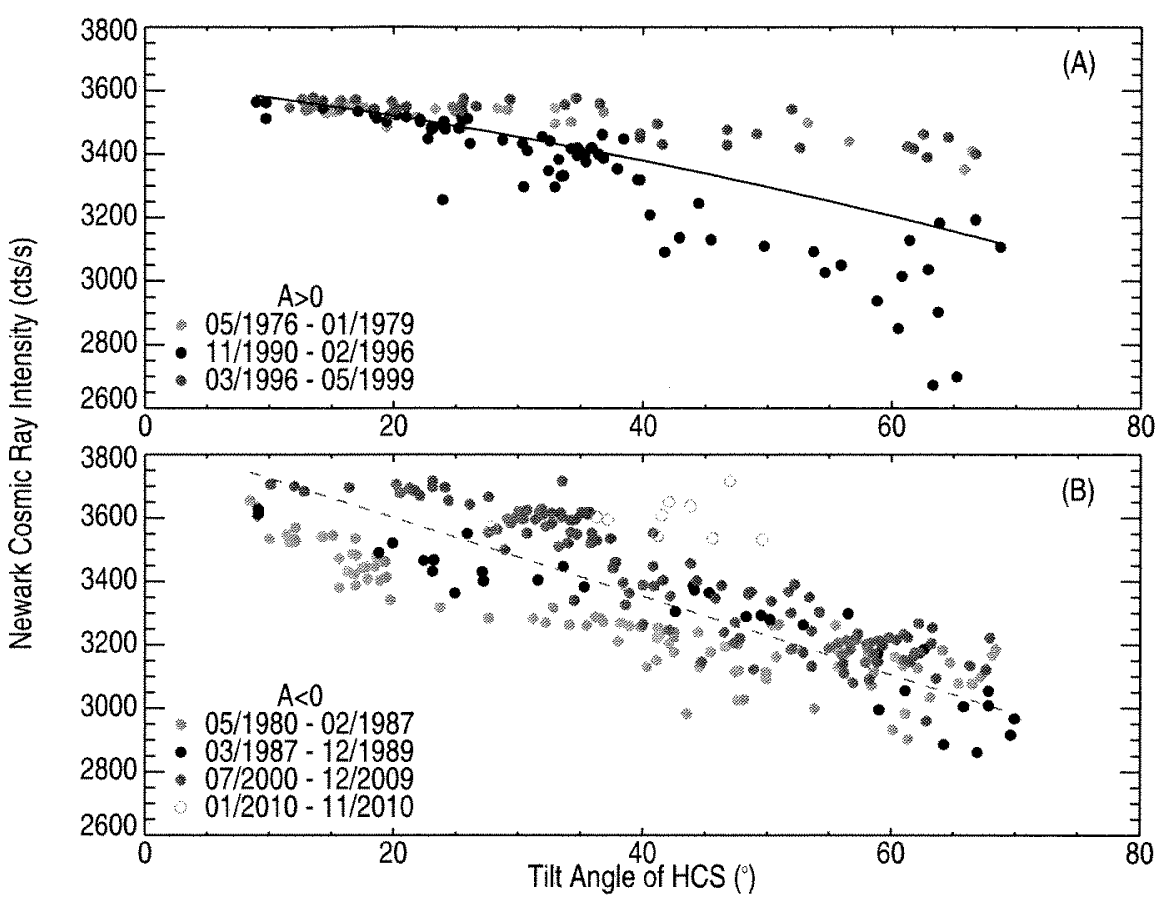

Fig. 2 Scatter plots, with regression lines, of 27 -day averages of the Newark neutron monitor counting rate vs. the "classic" tilt angle (which uses a line-of-sight boundary condition at the photosphere and includes a significant polar field correction) of the HCS from Wilcox Solar Observatory for (a) $A$ positive and (b) $A$ negative polarity epochs. Individual rise and fall intervals of the solar cycle are color coded. Intervals when $\alpha>70^{\circ}$ (undefined tilt angle) have been excluded from the plots

from cycle 21 , black filled points from cycle 22 , red filled points from cycle 23 , and blue open points from cycle 24 . Thus, the rising phase of cycle 21 , when $A>0$, is shown by the solid blue symbols in (a), while data for the declining phase, when $A<0$, are shown in (b). Note that the GCR intensity is a multi-valued function of the tilt angle for both polarity epochs, i.e., a single tilt angle corresponds to more than one GCR intensity. For example, during the A positive epoch in the 1990s, the recovery phase of modulation (black points in (a)) exhibited a much stronger dependence on tilt angle than did the onset phase (red points). The multi-valuedness of the functions in both Fig. 2(a) and (b) partially reflects the inadequacy of the tilt angle as a parameter for the drift effect. On the rise of a solar cycle, the large tilt angles in the plots $\left(\sim 60-70^{\circ}\right)$ will correspond to a shorter total length of the HCS than is the case for the decay phase. Thus, all else being equal, one would expect modulation to be greater for high tilt angles during the recovery of GCR intensity (resulting in the black points lying below the red points in (a)). Note in (b), however, that for low tilt angles, the red points corresponding to the decay of cycle 23 lie mainly above the solid blue points for the decay of cycle 21 while the blue circle data points for the rise of cycle 24 lie above the black points for the rise of cycle 22 . In these cases, the multi-valuedness of the GCR intensity vs. tilt relationship suggests that some factor other than drift is driving the modulation. We will pursue this point in greater detail below.

Figure 3 shows the Newark GCR intensity plotted against solar wind $B$ (27-day averages). In this case, the GCR intensity is more nearly a single-valued function, albeit with significant scatter, of solar wind $B$ for the rise and fall phases within a given polarity epoch. However, there is a clear difference in the response of the GCR intensity to changes in $B$ in $A$ positive and negative epochs. For low $B$ values $(\lesssim 6 \mathrm{nT}$ ) in $A$ positive epochs (Fig. 3(a)), 


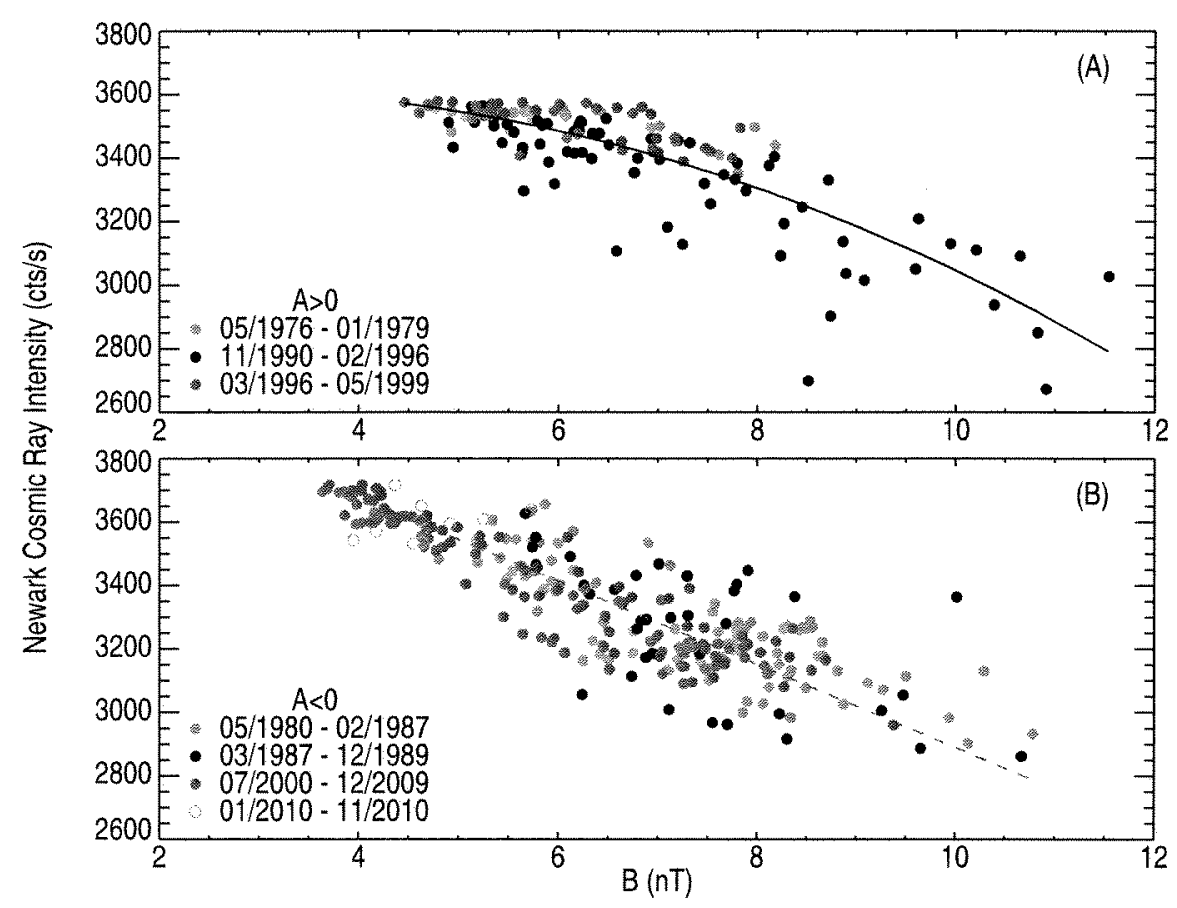

Fig. 3 Scatter plots, with regression lines, of 27-day averages of the Newark neutron monitor counting rate vs. 27-day averages of solar wind $B$ for $(\mathbf{a}) A$ positive and $(\mathbf{b}) A$ negative polarity epochs. Individual rise and fall intervals of the solar cycle are color coded. Rise portions include $\alpha>70^{\circ}$ intervals. The regression line in (a) is a second order fit

GCR intensity decreases more slowly as $B$ increases (cf., Wibberenz et al. 2002) than is the case for $A$ negative epochs (Fig. 3(b)).

This effect can also be seen in Figs. 4 and 5. In Fig. 4(a) and (b), we consider the GCR response to changes in $B$ for fixed ranges of the tilt angle in negative and positive polarity epochs, respectively. Note that in $A$ negative epochs (Fig. 4(a)), the slope is comparable over the full range of tilt angles. In contrast, during $A$ positive epochs (Fig. 4(b)), the response for low tilt angles is weak (neglecting the circled outlier in the correlation analysis) but increases rapidly with increasing tilt angle. Figure 4 is a direct demonstration of the suggestion by Wibberenz et al. (2001) that "for $A>0$ polarity epochs, the sensitivity of the CR response to variations in $B$ is partly controlled by the size of the tilt angle, $\alpha$." Figure 5 contains time lines of: (a) the tilt angle and Newark cosmic ray intensity (inverted, 27-day averages) for 1976-2009, and (b) solar wind $B$ (27-day averages) and Newark GCR intensity (inverted, 27-day averages) from 1965-2009. While the correlation coefficients between the parameters in the two panels are not greatly different (e.g., $r=0.80$ in (a) vs. 0.74 in (b) for 27-day averages from 1976-2009), the closer relationship of $B$ with the cosmic ray intensity for the common years in the plots (in comparison with the relationship between $\alpha$ and the cosmic ray intensity), both for phase and amplitude, is apparent. The weaker fidelity between the tilt angle and GCR intensity is noticeable on the rise of the odd-numbered solar cycles 21 and 23, an effect reported previously by Cliver and Ling (2001a) (see also Nagashima and Morishita 1980, and van Allen 2000), and is particularly evident during the decline of cycle 23 and the rise of cycle 24. It is instructive to bring the data in Fig. 5 to bear on the multi-valued nature of the relationship between the GCR intensity and the tilt angle in Fig. 2. Specifically, we note: (i) the large separation between the red and black points for tilt angles of $\sim 60^{\circ}$ in (a) corresponds to a $\sim 2.5 \mathrm{nT}$ difference in solar wind $B$ 

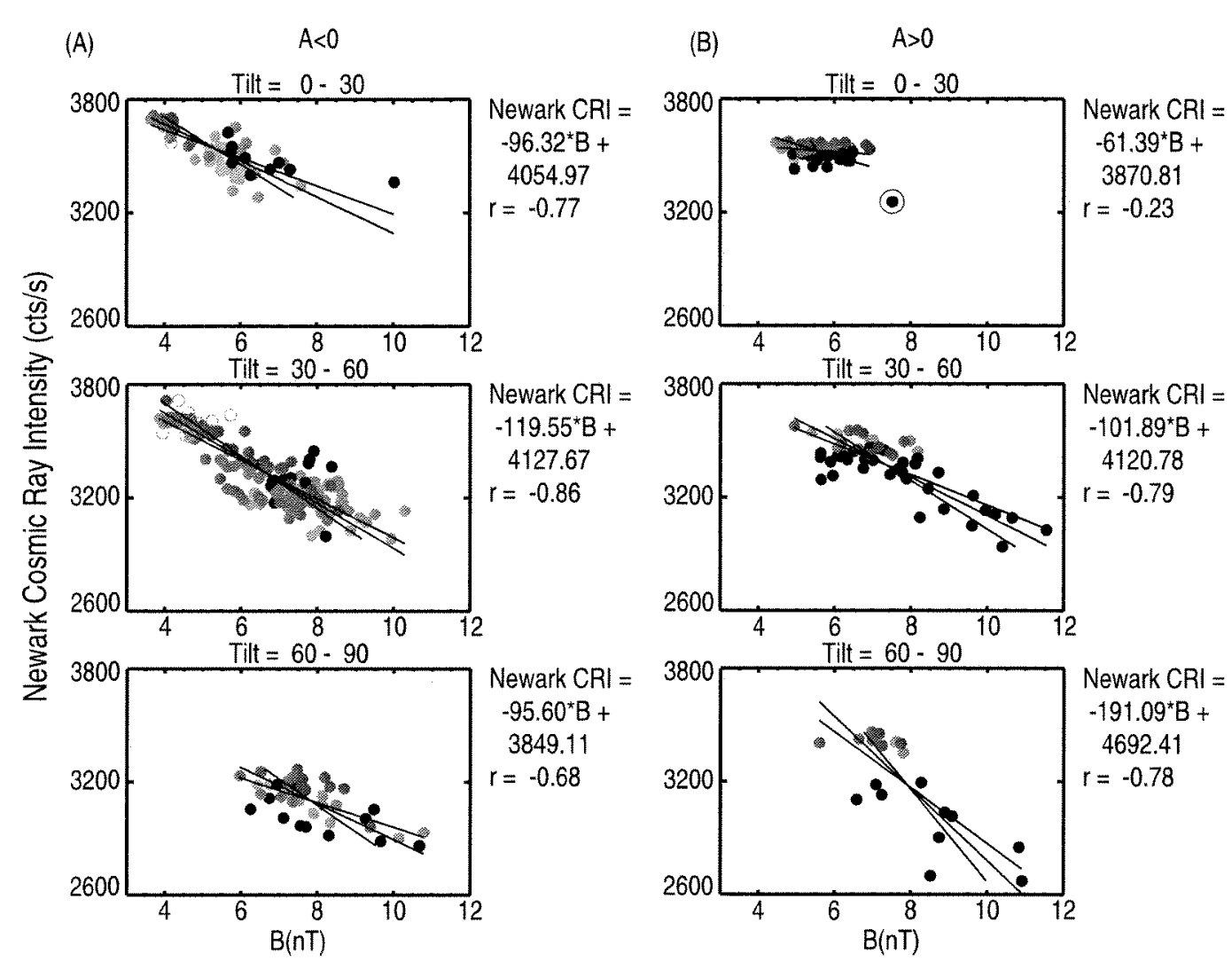

Fig. 4 (a) Scatter plots of the Newark neutron monitor counting rate (or cosmic ray intensity (CRI)) vs. solar wind $B$ for three ranges of the tilt angle during $A$ negative polarity epochs. The color coding is the same as in Figs. 3 and 4. (b) Scatter plots of the Newark CRI vs. solar wind $B$ for three ranges of the tilt angle during A positive polarity epochs. The circled outlier (Carrington Rotation 1856; 20 May-16 June 1992) in the top panel was not used in the correlation analysis

between 1998.5-1999.5 and 1991; (ii) the separation between the red and solid blue points for tilt angles of $\sim 30^{\circ}$ in (b) corresponds to a $\sim 2.5 \mathrm{nT}$ difference in solar wind $B$ between 2007 and 1984.5-1985.5; (3) and the separation between the blue circle and black points for tilt angles of $\sim 45^{\circ}$ in (b) corresponds to a $\sim 2.5 \mathrm{nT}$ difference in solar wind $B$ between $\sim 2010$ and 1988. In each case, the year with the larger value of $B$ had the lower associated GCR intensity, consistent with the notion that solar wind $B$ is the primary driver of GCR modulation at all phases of the 11 -yr cycle.

Figure 6 provides support for the idea that tilt angle variation does not drive modulation at solar minimum as is commonly thought to be the case (e.g., Potgieter and Le Roux 1994; Jokipii and Wibberenz 1998; Ferreira and Potgieter 2004). Figure 6(a) shows that while the lowest value of the annually-averaged tilt angle was essentially constant $\left(\sim 15-20^{\circ}\right)$ at the last four solar minima (actually increasing slightly in 2009 relative to the three previous minima), the corresponding cosmic ray intensity was significantly $(\sim 5 \%)$ higher during the minimum in 2009. As shown in Fig. 6(b), the marked increase in cosmic ray intensity at Newark during this minimum was accompanied by a significant decrease (from $\sim 5.4 \mathrm{nT}$ to $\sim 3.9 \mathrm{nT}$ ) in solar wind $B$, relative to the three previous minima.

A final point on the role of drift on the 11-year variation of GCR intensity-in basic drift theory (Jokipii and Thomas 1981; Kóta and Jokipii 1983) the tilt angle has the same or at least a non- $A$ dependent evolution from cycle-to-cycle. Figure 7 shows that 


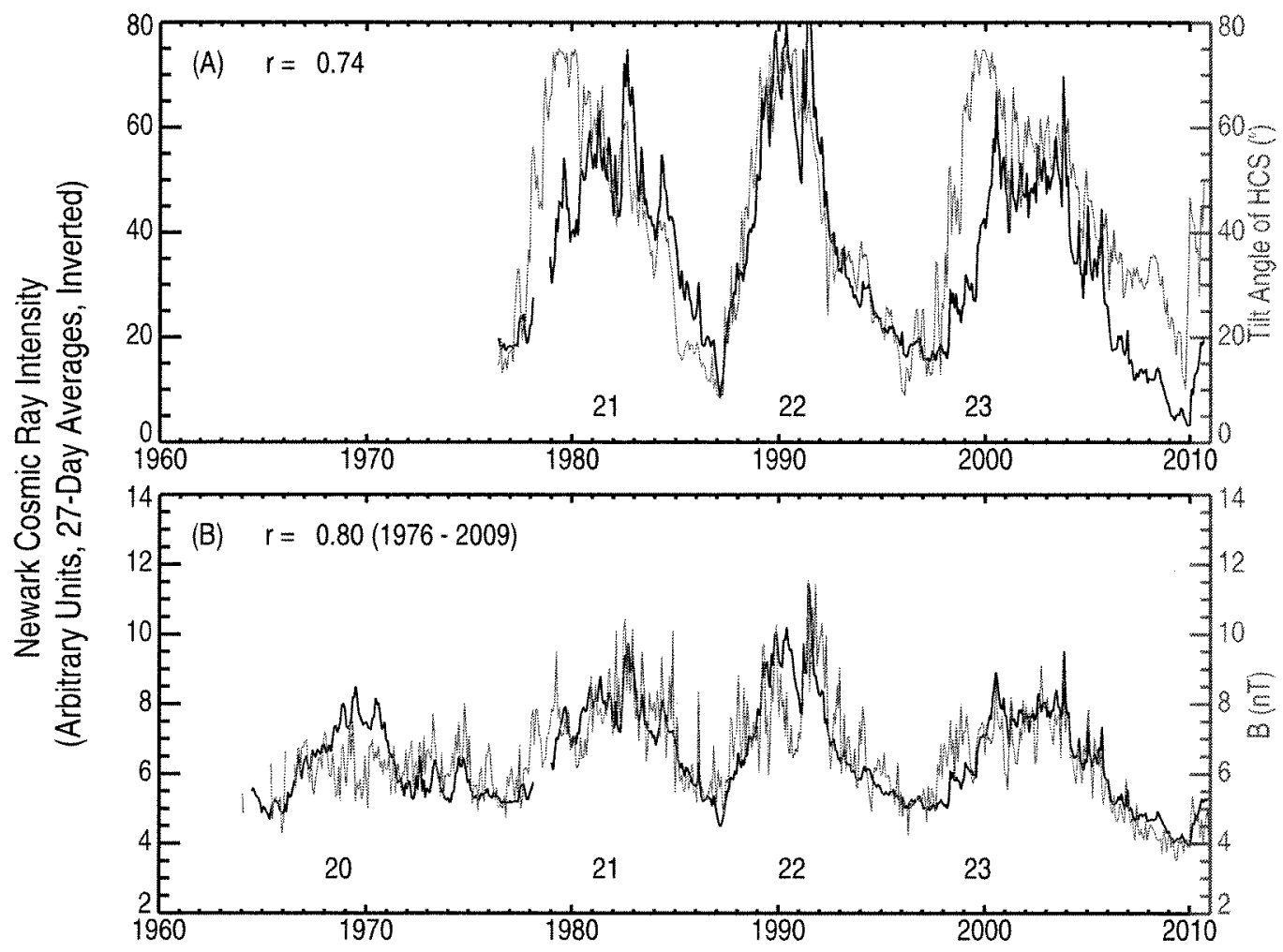

Fig. 5 (a) Tilt angle of the HCS (red) and Newark neutron monitor counting rate (black; 27-day averaged, inverted, arbitrary units) from 1976-2009. (b) Solar wind $B$ (red;27-day averaged) and Newark neutron monitor counting rate (black; 27-day averaged; inverted, arbitrary units) from 1976-2009

while that this assumption may be true for the rise of the cycle, it may not be true for the decline (Cliver 1993; Cliver et al. 1996; Cliver and Ling 2001a). In cycle 22, the tilt angle declined more rapidly in comparison with cycles 21 and 23 . A relatively rapid decrease also appears to have occurred during cycle 20 (Hundhausen et al. 1980; Cliver 1993). A longer run of observations is required to establish the reality/regularity of this cycle-to-cycle variation in the tilt angle and to assess any associated systematic effects on the shapes of modulation cycles. The tilt angle record through 2010 indicates that thus far cycle 24 is evolving differently from the previous three cycles, with tilt angles $\sim 45^{\circ}$ reached more rapidly than in the past.

\subsection{How Does the Sun Transmit Its Message of Increased Magnetic Field to the Heliosphere?}

To answer this question, we will make use of a decomposition of the solar wind into its three principal components-slow solar wind (SSW), high-speed streams (HSSs), and transient structures including CMEs and post-shock flows, and an additional category for intervals when the flow type could not be determined, e.g., due to data gaps. This decomposition was made by Richardson et al. $(2000,2001,2002)$ for the years 1972-2000 and has been updated through 2009, and extended back to 1965, for the present study. The decomposition is based on OMNI solar wind data and ancillary data from solar energetic particle detectors and neutron monitors, as discussed in Richardson et al. (2000). Figure 8 shows the contribution of each of the three flow types to annual averages of solar wind $B$ for 1965-2009, with 


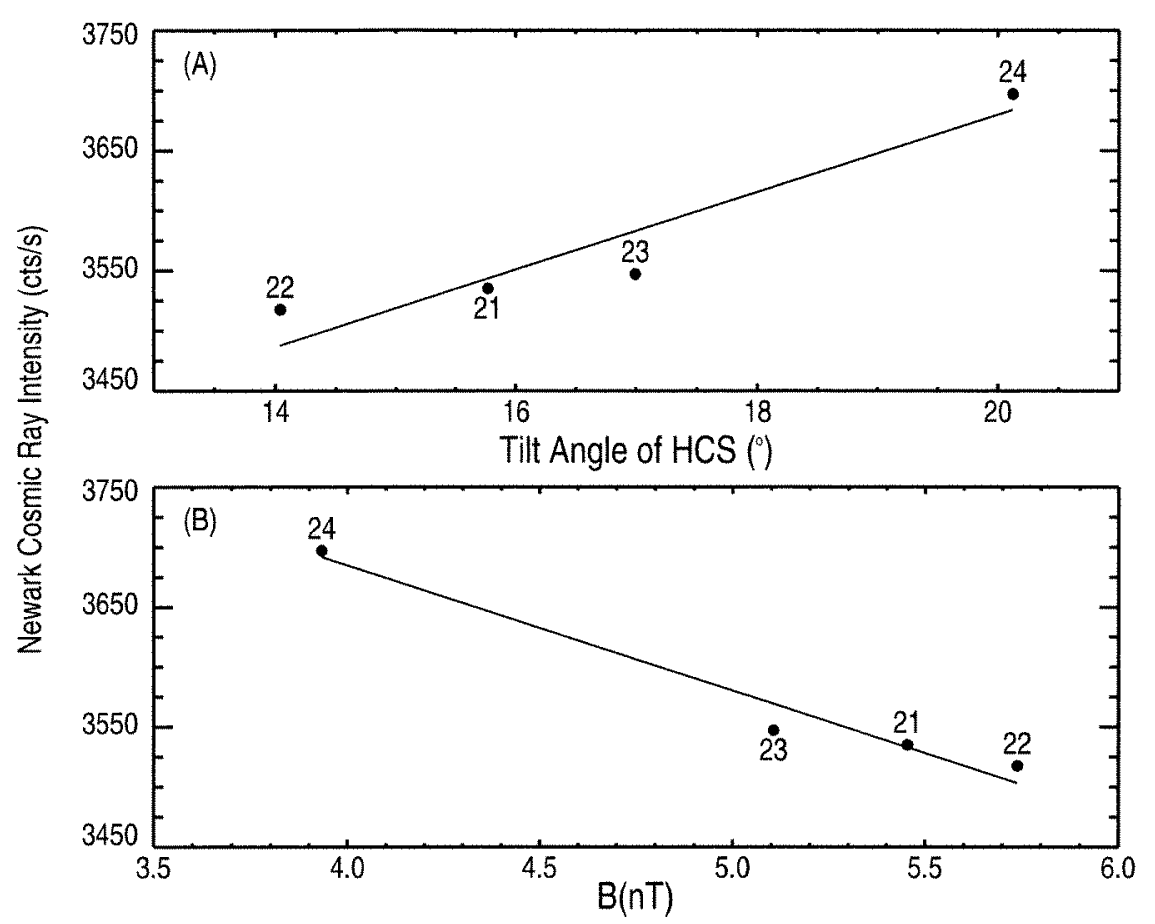

Fig. 6 (a) Yearly-averages of Newark neutron monitor counting rate vs. yearly-averages of the tilt angle of the heliospheric for the last four solar minima. (b) Yearly-averages of Newark neutron monitor counting rate vs. yearly-average of solar wind $B$ for the last four solar minima. The minima are years with lowest average $B$ and $\alpha$ values; for the 1986 and 1996 minima, the cosmic ray maxima occurred the following year, being higher by $15 \mathrm{cts} \mathrm{s}^{-1}$ and $17 \mathrm{cts} \mathrm{s}^{-1}$, respectively, than the plotted values

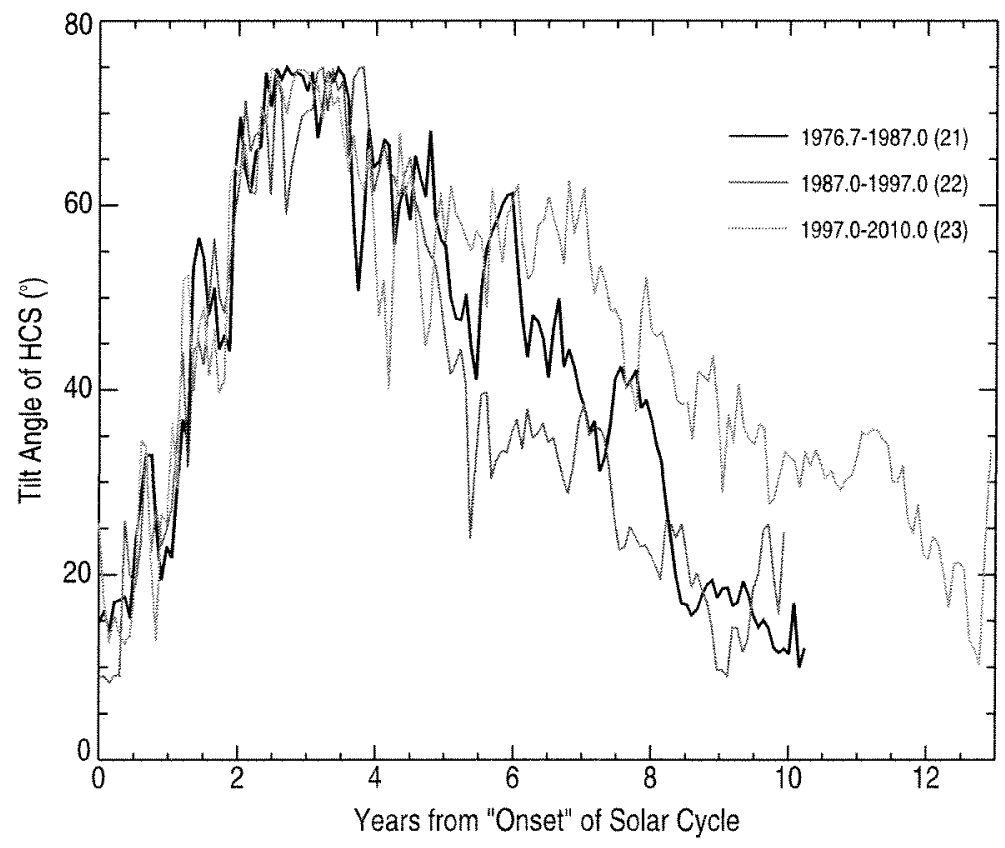

Fig. 7 Superposed epoch plot showing the evolution of the tilt angle of the HCS during each of the last three solar cycles (updated from Cliver and Ling 2001a). The zero epoch was adjusted to obtain best agreement for the rise phases of the three cycles 


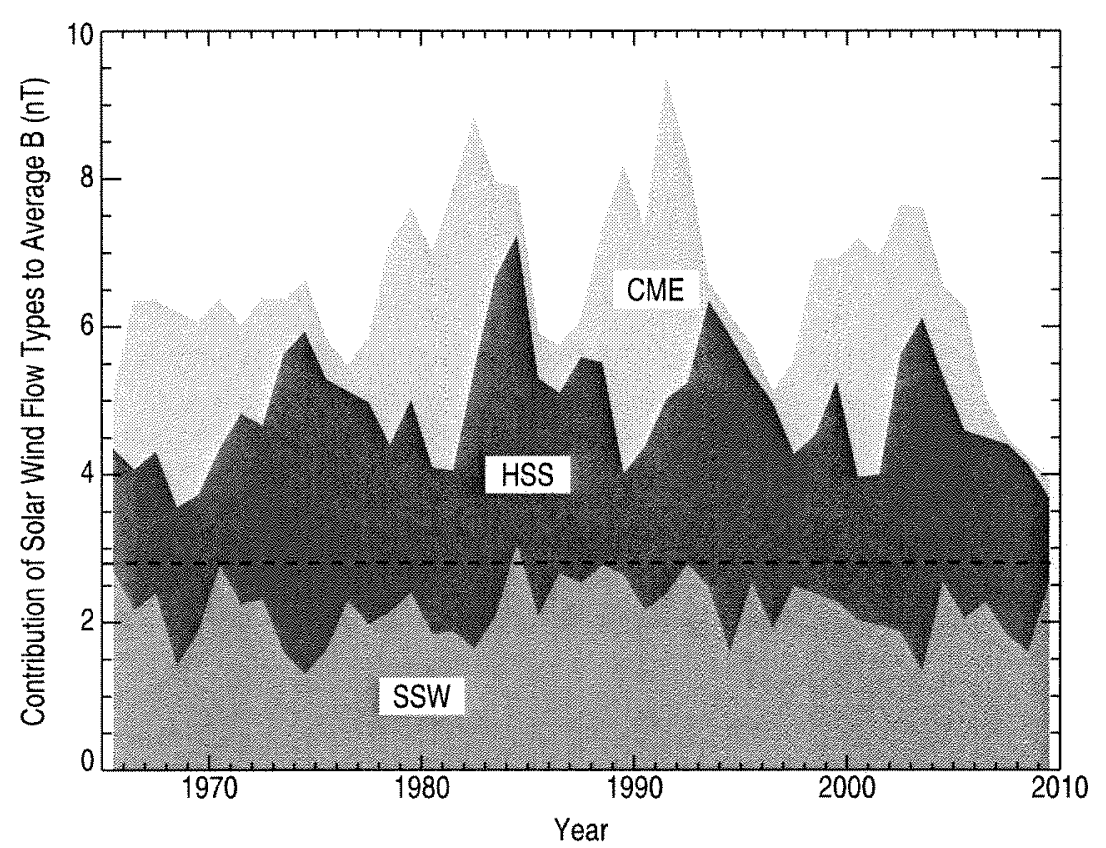

Fig. 8 Cumulative distribution graph showing the contribution of SSW, HSSs, and CMEs to average $B$ from 1965-2009 (adapted from Cliver and Ling 2010). The dashed line is drawn at $2.8 \mathrm{nT}$, the revised position of the "floor" in the solar wind

the contribution from the unclassified intervals distributed proportionately (according to the time Earth was immersed in SSW, HSSs, and CMEs during the year) among the three basic types. Note that the $y$-axis in Fig. 8 is not the average $B$ in each flow type, although the sum of the three contributions adds up to average $B$ for each year. Rather it is what average $B$ would have been for a given flow type in the absence of the other two types (setting their contributions equal to zero and dividing the sum of hourly average $B$ values in, e.g., CME flows, by the total number of hours of observations during a given year). We will refer to these contributions to $B$ as $B_{\mathrm{SSW}}, B_{\mathrm{HSS}}$, and $B_{\mathrm{CME}}$.

Figure 8 shows that $B_{\mathrm{Ssw}}$ does not vary strongly with the solar cycle; it has a mean value of $\sim 2.2 \pm 0.4 \mathrm{nT}$ over the 44-yr interval. $B_{\mathrm{HSS}}$ peaks on the decline of the cycle in accord with the well-known tendencies for coronal holes and recurrent geomagnetic storms to be prominent during that phase of the cycle (e.g., Crooker and Cliver 1994). It appears that $B$ from HSSs dominated modulation events ("minicycles") on the decline of the solar cycle in 1974 (Hewish 2001; Cliver et al. 2003), 1984, and 1994, when $B_{\mathrm{CME}}$ is relatively small. Note that at the solar minima in 1976,1986, 1996, and 2009, CMEs make a relatively small contribution to $B$. Thus the $\sim 5 \%$ increase in the Newark neutron monitor counting rate in 2009 above the average for the three previous minima (Fig. 6) is attributed to the decrease in $B_{\text {HSS }}$ during this year. The decrease in $B_{\text {HSS }}$ from $\sim 2.8 \mathrm{nT}$ during $1976 / 1986 / 1996$ to $\sim 1.2 \mathrm{nT}$ in 2009 accounts for the $\sim 1.5 \mathrm{nT}$ drop in $B$ during these periods (from $\sim 5.4 \mathrm{nT}$ to $\sim 3.9 \mathrm{nT}$ ). The contribution to $B$ from CMEs tracks the solar cycle (Webb and Howard 1994), and essentially disappears at solar minimum. Figure 8 shows that CMEs are primarily responsible for increasing $B$ at solar maximum, and that HSSs are principally responsible for $B$ variation at solar minimum as well as during modulation minicycles on the approach to minimum. Figure $9(\mathrm{a}, \mathrm{b}, \mathrm{c})$ shows scatter plots of GCR intensity vs. $B_{\mathrm{CME}}, B_{\mathrm{HSS}}$, and $B_{\text {SSW }}$, respectively. The strongest anti-correlation is for CMEs; in fact, for SSW, there is a weak positive correlation. 


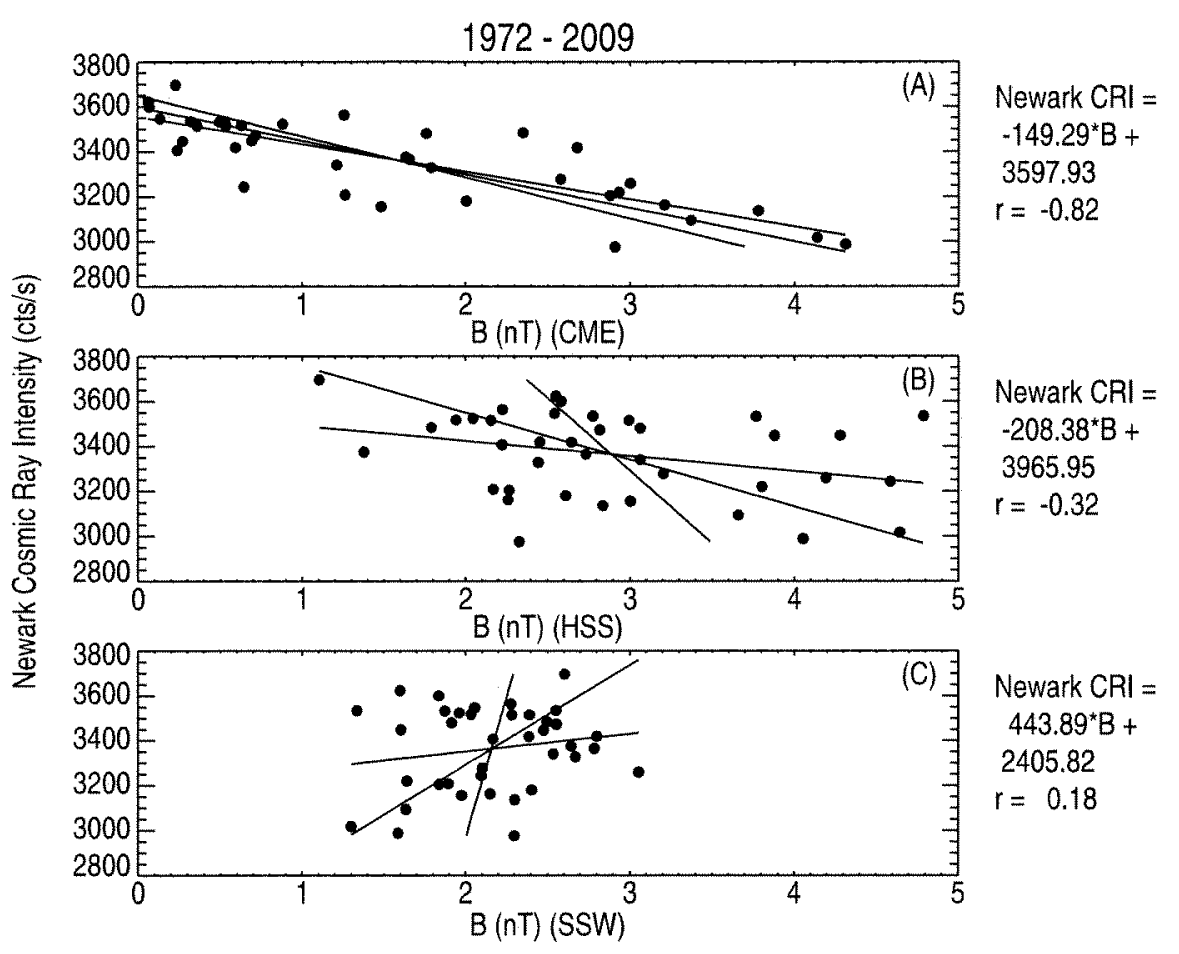

Fig. 9 Scatter plots, with regression lines, of Newark GCR intensity vs. (a) $B_{\mathrm{CME}}$, (b) $B_{\mathrm{HSS}}$, and (c) $B_{\mathrm{SSW}}$

\subsection{A Paradox Involving GCR Electrons at the Recent Solar Minimum}

Heber et al. (2009) noted that the modulation amplitude of GCR electrons during 2008 exceeded the level reached during the mid-1990s minimum by $30-40 \%$. In comparison, the modulation amplitude for the Newark neutron monitor in 2009 increased by only $\sim 4 \%$ over the 1997 minimum. Modulation models predict that at true minimum the normalized count rates of both GCR electrons and protons should be equal (Potgieter et al. 2001). The failure of the modulation amplitude of the protons to reach a value commensurate with that for electrons is attributed (Heber 2010) to the fact that the tilt angle dropped below $15^{\circ}$ for only 3 consecutive rotations in 2009, vs. 8 in 1995-1996. The lowest yearly (for any 13 consecutive rotations) average of the tilt angle during the mid-1990s was $\sim 13^{\circ}$ [August 1995-June 1996] vs. $19^{\circ}$ for the corresponding interval during the first decade of the 21 st century [December 2008-November 2009]. Correlations based on cycles 21-23 (Fig. 10) show that the decrease of $\sim 1.2 \mathrm{nT}$ between the average values of $B$ for 1997 and 2009 is far too small to account for a 30-40\% increase in the modulation amplitude of GCR protons at Newark (a) and that the corresponding increase of $\sim 5^{\circ}$ in the tilt angle is far too small to negate such an increase (b). For example, as shown in Fig. 10(b), the average response of the Newark neutron monitor to a $10^{\circ}$ change in the tilt angle is only $\sim 3 \%$.

\subsection{Long-Term Modulation}

\subsubsection{Emerging Consensus on Long-Term Reconstructions of B}

Long-term reconstructions of the solar wind magnetic field, once contentious (e.g., Lockwood et al. 2006; Svalgaard and Cliver 2006; McCracken 2007), have recently reached a 


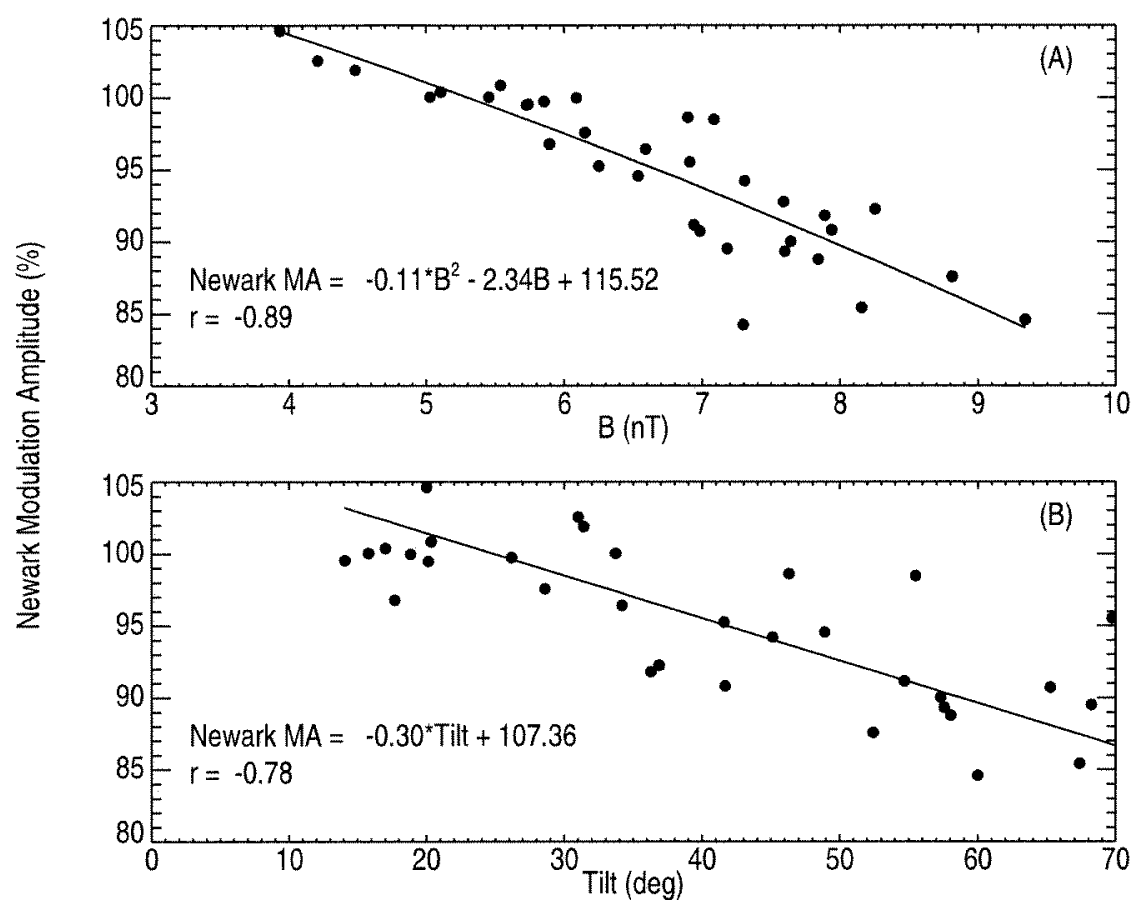

Fig. 10 (a) Scatter plot (with regression line) of yearly values of the GCR modulation amplitude (MA) measured by the Newark neutron monitor from 1976-2009 vs. solar wind $B(1987=100 \%)$. (b) Scatter plot (with regression line) of yearly values of the GCR modulation amplitude measured by the Newark neutron monitor from $1976-2009$ vs. the tilt angle of the $\operatorname{HCS}(1987=100 \%)$

consensus for the 20th century. In Fig. 12 of their recent paper, Svalgaard and Cliver (2010) show the evolution and, for the most part, resolution between the discordant geomagneticbased reconstructions of Lockwood et al. (1999) and Svalgaard and Cliver (2005). Furthermore, in their Fig. 14, they show that the Steinhilber et al. (2010; cf., Webber and Higbie 2010a, 2010b; Webber et al. 2010) ${ }^{10}$ Be-based reconstruction of $B$, which superseded and extended that of McCracken (2007), is now in general agreement with the geomagneticbased reconstructions for the last $\sim 100$ years.

In Fig. 11, we use the regression relationship between the modulation amplitude of the GCRs measured by the Newark neutron monitor and annual averages of solar wind $B$ $(r=0.89$ ) from Fig. 10(a) and the reconstruction of $B$ from 1835-2009 obtained by Svalgaard and Cliver (2010) to reconstruct the modulation amplitude of $\sim 2 \mathrm{GV}$ protons for this interval and compare it with observations from 1963-present. Despite the gross simplicity of this approach, the reconstructed GCR modulation amplitude matches observations fairly well, except for cycle 20 (indicated by the blue oval). The good agreement within the oval between the reconstructions of the amplitudes based on actual $B$ and on IDV-based $B$ (from Svalgaard and Cliver 2010) indicates that the measurements of $B$ during the early space age are sound and that the cause of the cycle 20 anomaly does not lie with erroneous $B$-values (as suggested, e.g., Belov 2000). Wibberenz et al. (2002) were able to model cycle 20 with reasonable fidelity using the observed $B$ and the longer GCR recovery time ( $T \sim 100$ days) characteristic of $A$ negative cycles and solar maximum intervals. 


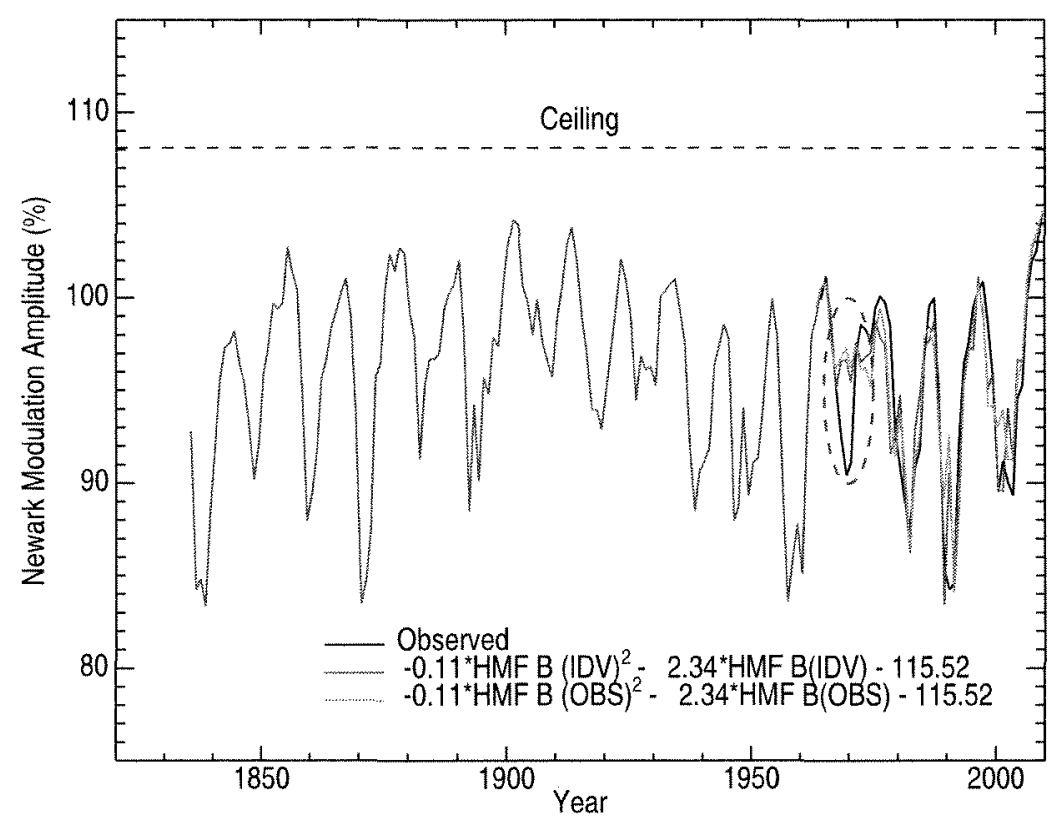

Fig. 11 Reconstruction of annual values of the GCR modulation amplitude measured by the Newark neutron monitor based on the regression relationship in Fig. 10(a) and derived using: (a) observed solar wind $B$ (blue line), and (b) IDV09-based B (red line) from Svalgaard and Cliver (2010). The observed $\sim 2$ GV modulation amplitude (black line) is shown for comparison. The dashed blue oval highlights the anomalous behavior of solar cycle 20 and the dashed black line indicates the ceiling in the cosmic ray intensity corresponding to a floor of $\sim 2.8 \mathrm{nT}$

\subsubsection{A Floor in the Solar Wind Magnetic Field: Implications for GCR Modulation}

Recently, Cliver and Ling (2010) have argued that a floor exists in the solar wind magnetic field strength of $\sim 2.8 \mathrm{nT}$ (cf., Svalgaard and Cliver 2007). They attribute the floor to a constant baseline open flux of $\sim 8 \times 10^{13} \mathrm{~Wb}$ associated with the slow solar wind. Cliver and Ling suggest that the floor corresponds to a ground-state of the solar wind, achieved in the absence, or near absence, of the solar cycle, i.e., during periods such as the Maunder Minimum (Eddy 1976). Figure 12 shows that the existence of a floor in $B$ implies a floor in the modulation potential $(\Phi)$ of $\sim 100 \mathrm{MV}$. Moreover, the regression equation from Fig. 10(a) and dashed line in Fig. 11 show that a floor in $B$ of $\sim 2.8 \mathrm{nT}$ (or $\Phi$ of $\sim 100 \mathrm{MV}$ ) implies a ceiling in the cosmic ray intensity $(\sim 108 \%$ for Newark; $1987=100 \%)$. In a sense, the floor results in a permanent modulation of the local interstellar spectrum at any given energy or rigidity.

The reconstruction of solar wind $B$ by Steinhilber et al. (2010) extends back over the last $\sim 10^{4}$ years (Fig. 13). During this interval there are nine periods, beginning with the Spörer Minimum (Eddy 1976) from 1420-1570, where $B$ dropped to zero, violating the notion of a floor. We will discuss these extreme dips further in Sect. 3.

\section{Conclusion}

\subsection{Summary}

In this study of solar and solar wind drivers of 11 -yr and long-term modulation of the $\sim 2 \mathrm{GV}$ galactic cosmic ray proton intensity, we have found the following: 


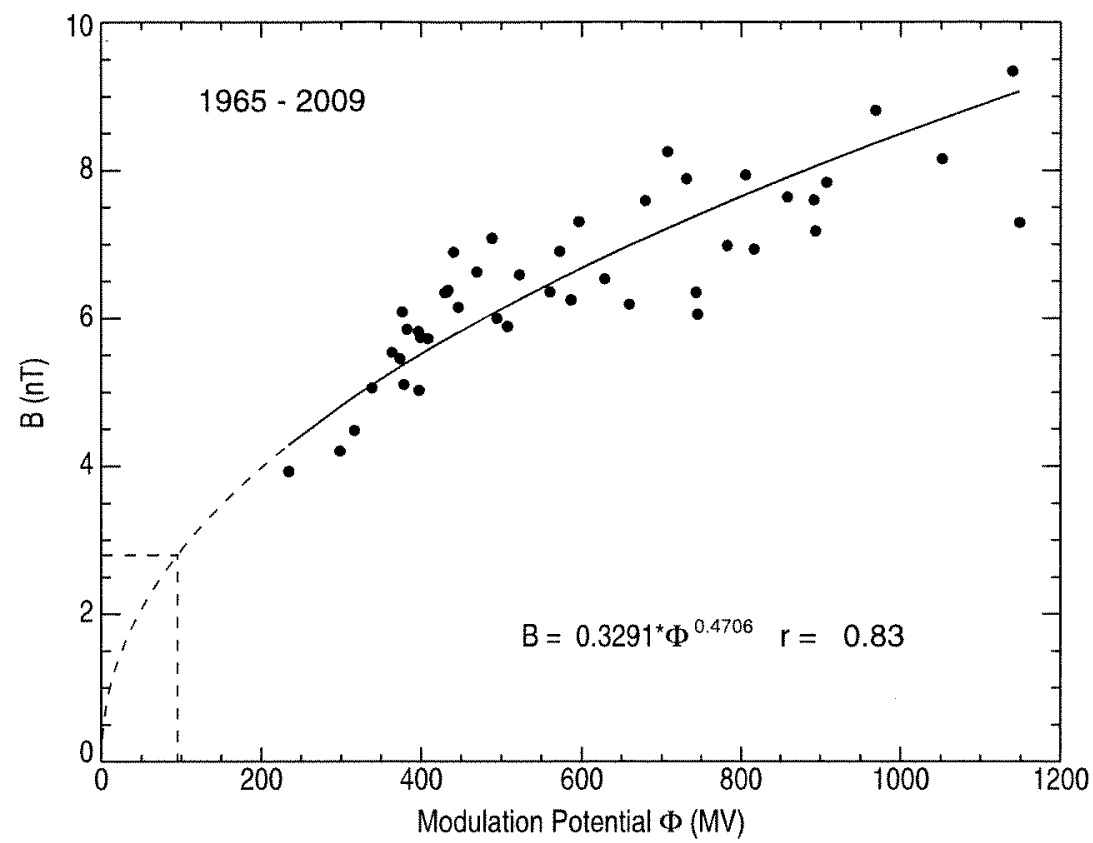

Fig. 12 Solar wind $B$ as a function of the modulation potential (after Steinhilber et al. 2010); the $\Phi$ values were taken from: http:/www.faa.gov/data research/research/med humanfacs/aeromedical/medial caribmex.zip (see Fig. 2 in Steinhilber et al. 2010). A floor of in $B$ of $\sim 2.8$ nT implies a minimum $\Phi$ of $\sim 100 \mathrm{MV}$

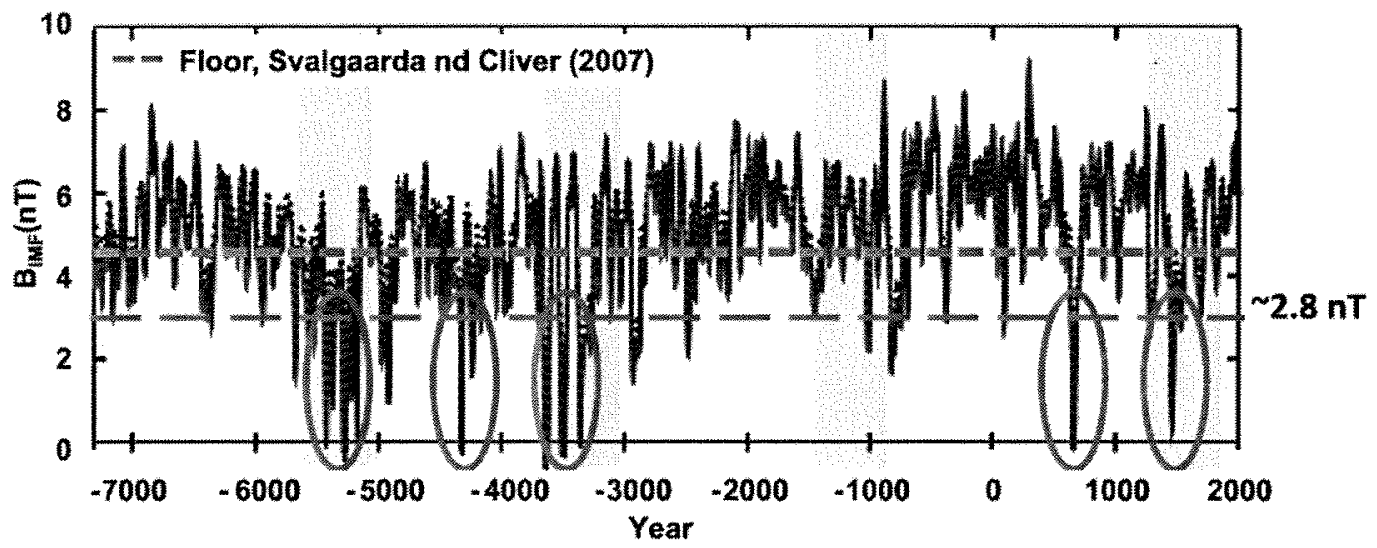

Fig. 13 Reconstruction of $B$ (40-yr running means) based on ${ }^{10} \mathrm{Be}$ concentration in ice cores for the last $\sim 10^{4}$ years (adapted from Steinhilber et al. 2010). The dashed green line drawn at $\sim 4.6 \mathrm{nT}$ represents the value of the floor reported by Svalgaard and Cliver (2007) and the dashed red line drawn at $\sim 2.8 \mathrm{nT}$ indicates the revised floor (Cliver and Ling 2010). The red ovals encompass sharp drops to $B$-values $\lesssim 0 \mathrm{nT}$

(1) GCR intensity is a multi-valued function of the tilt angle of the heliospheric current sheet, both for different phases (rise and fall) of the solar cycle within a given $A$ positive epoch, and for the same phase of different solar cycles within $A$ negative epochs. This multi-valuedness is due, in part, to the inadequacy of the tilt angle as a parameter for drift modulation.

(2) GCR intensity is roughly a single-valued function of solar wind $B$ within a given polarity epoch, but the function differs between $A$ positive and $A$ negative epochs. In 
particular, GCR intensity has a weaker response to changes in $B$ near the onset of the modulation cycle (i.e., for low $B$ values and tilt angles) in $A$ positive epochs.

(3) The record high cosmic ray intensities observed in 2009 resulted from a reduction in $B$ (from $\sim 5.4 \mathrm{nT}$ to $\sim 3.9 \mathrm{nT}$ ) in comparison with the 1986 minimum, rather than from a reduction in the tilt angle (which actually increased slightly in 2009 , to $\sim 14^{\circ}$, vs. $\sim 20^{\circ}$ in 1986).

(4) The tilt angle $(\alpha)$ evolution may be systematically different in even- and odd-numbered cycles. In cycles 20 and $22, \alpha$ appeared to decay more quickly following solar cycle maximum than it did in cycles 21 and 23.

(5) The Sun's message of changing magnetic field is transmitted to the heliosphere primarily by CMEs at solar maximum and by HSSs at solar minimum.

(6) The cosmic ray intensity is anti-correlated $(r=-0.82)$ with the contribution of CMEs to solar wind $B$ (based on annual averages), weakly anti-correlated $(r=-0.32)$ with the contribution of HSSs to $B$, and essentially uncorrelated with the SSW contribution.

(7) The $\sim 30-40 \%$ increase in the modulation amplitude of $\sim 2.5 \mathrm{GV}$ cosmic ray electrons during the recent cycle minimum suggests that the intensity of $\sim 2 \mathrm{GV}$ protons should have increased by a comparable amount (Heber et al. 2009). However, such an increase for protons would have been inconsistent with a close relationship between the Newark cosmic ray intensity and solar wind $B$ from 1976-2009. Moreover, based on the relationship between the Newark neutron monitor count rate for this same interval and the tilt angle of the HCS, it is difficult to attribute the observed absence of a $\sim 35 \%$ increase in the $\sim 2 \mathrm{GV}$ proton count rate to a larger tilt angle.

(8) Long-term reconstructions of solar wind $B$, both those based on geomagnetic data and those inferred from cosmogenic nuclei data from tree rings and ice cores, are in good agreement for the 20th century.

(9) The close relationship between solar wind $B$ and GCR intensity can be used to reproduce the modulation curve with reasonable fidelity during the space age, with the exception of cycle 20 . This relationship can then be combined with the validated longterm reconstruction of $B$ to confidently extend the modulation curve back to at least $\sim 1900$.

(10) A recently proposed "ffloor" in solar wind $B$ of $\sim 2.8$ nT implies a minimum (permanent baseline) modulation potential of $\sim 100 \mathrm{MV}$ that results in a ceiling in the cosmic ray intensity which lies below the local interstellar (LIS) spectrum at any given energy/rigidity. The notion of a floor in $B$ is at variance with a recent ${ }^{10} \mathrm{Be}$-based reconstruction (Steinhilber et al. 2010) that shows several episodes when B was $\sim 0$ nT during the past $\sim 10^{4}$ years.

\subsection{Discussion}

While it is generally accepted that barrier modulation is dominant at solar maximum, drift effects are thought to control modulation away from maximum. The solar minimum year of 2009 provided a controlled experiment on this point. During 2009, the tilt angle was essentially constant in comparison with previous minima while solar wind $B$ dropped, and the $\sim 2$ GV cosmic ray intensity rose, significantly. Thus it appears that diffusion, driven by the minimum-to-minimum variation in $B$, is the primary modulation process at this phase of the solar cycle as well. This picture is supported by the multi-valued relationship between GCR intensity and the tilt angle and the more nearly single-valued relationship between GCR intensity and $B$, for both polarity epochs, from 1976-present.

The Sun sends its message of increased magnetic field (reduced diffusion coefficient) to the heliosphere primarily via CMEs at maximum, with high-speed streams playing an 
increasing role as the cycle declines (e.g., during the mini-cycles in 1974, 1984, and 1994). At solar minima, when CMEs occur infrequently, HSSs from polar coronal holes (Wang and Sheeley 1994) are the main source of cycle-to-cycle variation in $B$ that drives modulation at these times. The high Newark neutron monitor counting rate in 2009, compared to the average rate for 1976, 1986, and 1996, resulted from the drop in the average contribution of HSSs to $B$ from $\sim 2.8 \mathrm{nT}$ in $1976 / 1986 / 1996$ to $\sim 1.2 \mathrm{nT}$ in 2009 (thus accounting for the drop in $B$ from $\sim 5.4 \mathrm{nT}$ to $\sim 3.9 \mathrm{nT}$ ). The drop in $B_{\mathrm{HSS}}$ in 2009 is a direct reflection of the $\sim 45 \%$ decrease in the solar polar field strength between the minima preceding Cycles 23 and 24 (Svalgaard et al. 2005; Cliver and Ling 2010). Cliver and Ling (2010) equated the SSW with a floor, or constant baseline level, in the solar wind. Such a floor will not contribute to either the $11-\mathrm{yr}$ or long-term variation of GCR intensity.

The effects of large-scale curvature and gradient drifts are most notable at the onset of the modulation cycle in $A$ positive epochs (the rise of odd-numbered solar cycles) when GCRs respond weakly to increases in $B$ (and tilt angle). The weaker response of the GCR intensity to changes in $B$ at such times is attributed to the drift-induced preference for positively charged particles to approach the inner heliosphere from the poles at these times (Jokipii and Thomas 1981) and the relative confinement of CMEs to low latitudes at the onset of the solar (modulation) cycle (Hundhausen 1993; Gopalswamy et al. 2010). As the cycle develops, CMEs form at progressively higher latitudes. Large broad CMEs originating at latitudes above $\sim 30-40^{\circ}$ can encompass the polar regions and begin to "close the shell" in the inner heliosphere (McDonald et al. 1993; Cliver et al. 1993; Cliver and Ling 2001a; Lara et al. 2005). As demonstrated by Potgieter and Le Roux (1994), particle inflow from over the poles of the Sun during $A$ positive cycles is more rapid than inflow along the wavy neutral sheet during the corresponding phase of $A$ negative cycles. Thus GCR recovery times are shorter during $A$ positive cycles (Wibberenz et al. 2002). As a result, the onset of strong modulation is delayed at the onset of odd-numbered solar cycles until the polar regions are affected.

Thus far during the current cycle 24 , the GCR intensity vs. tilt angle relationship in Fig. 5(a) deviates significantly from that observed two cycles earlier on the rise of cycle 22. The unusually rapid rise of the tilt angle in 2010 is likely related to the weaker polar fields of the recent minimum (Svalgaard et al. 2005; Wang et al. 2009), which are eroding as the new cycle develops and the equatorial dipole gains strength. Note that despite the recent deviation in the GCR intensity vs. $\alpha$ relationship, the relationship between solar wind $B$ and GCR intensity (Figs. 3 and 5(b)) is largely unchanged by the data for the onset of the new cycle.

The quadratic relationship in Fig. 10(a) between the GCR intensity and $B$ for both polarity epochs reflects the delayed response of GCR intensity to the onset of modulation in $A$ positive cycles. The weaker response of GCRs to changes in low values of $B$ may also be due in part to the weaker (relative to CMEs) "cosmic-ray-effectiveness" of the high-speedstreams and CIRs (e.g., Burlaga et al. 1991, 1993; Cliver et al. 2003) which dominate the variable (non-floor) component of the solar wind at minimum (Cliver and Ling 2010).

Given the global nature of modulation, it is remarkable that a reconstruction based on a single parameter $(B)$ can largely reproduce the observed GCR intensity for the past $\sim 35$ years. The failure to do so during cycle 20 appears to be a relatively rare occurrence. Figure 5 in Svalgaard and Cliver (2005) reveals only one other cycle (14) in the last 130 years where $B$ was similarly disconnected from the sunspot number (and presumably cosmic ray intensity). Our $B$-based reconstruction of GCR intensity back to 1835 only applies to $\sim 2$ GV protons. As pointed out by Ferreira and Potgieter $(2003,2004)$, such a simplified 
approach does not work at all energies. A compound model involving both drift and diffusion is required to reproduce modulation curves at lower rigidities, as well as to simulate charge-dependent effects at higher energies.

The recent convergence of reconstructions of solar wind $B$ based on both geomagnetic and cosmogenic data gives confidence that we can go further back in time via ice core analysis. The deep minima (with $B \sim 0 \mathrm{nT}$ ), which stand out on the long-term reconstruction of Steinhilber et al. (2010), raise fundamental questions: (1) Can the solar wind go away? or (2) Can an unmagnetized solar wind exist? The revised floor value of $\sim 2.8 \mathrm{nT}$ recently suggested by Cliver and Ling (2010) accommodates the bulk of the low values in the Steinhilber et al. (2010) reconstruction of $B$ for the past $\sim 10^{4}$ years, except for the 9 sharp dropouts in which $B$ goes to zero and a comparable number of dips to $\sim 2 \mathrm{nT}$. A floor in solar wind $B$ implies a permanent modulation that results in a ceiling in the cosmic ray intensity below the local interstellar spectrum.

The conundrum of the large increase in the intensity of GCR electrons at the recent minimum will need to be resolved. There are three possibilities to explain the discrepancy between the electron and proton intensities: (1) the theory is incorrect/incomplete, i.e., electron and proton normalized count rates are not equal at minimum; or (2) the observation of the electron intensity, or its interpretation (e.g., rigidity value), is in error; or (3) the quantitative difference in $B$ between the last two minima $(\sim 1.2 \mathrm{nT})$ was large enough to effect a qualitative difference in the modulation.

In closing, we note one point in which all at the meeting were in agreement. The recent extension (by subtraction) of the Sun's dynamic range of activity makes this an exciting time to study the Sun and its impact on cosmic rays.

Acknowledgements It is a pleasure to thank Jürg Beer, Bernd Heber, Randy Jokipii, Jozsef Kota, Frank McDonald, Harm Moraal, and Rudolf von Steiger for organizing this timely and stimulating workshop. In addition, we are grateful to Marius Potgieter for constructive criticism. A.G.L. acknowledges support from AFRL contract FA8718-05-C-0036.

\section{References}

A. Belov, Space Sci. Rev. 93, 79 (2000)

L.F. Burlaga, F.B. McDonald, N.F. Ness, R. Schwenn, A.J. Lazarus, F. Mariani, J. Geophys. Res. 89, 6579 (1984)

L.F. Burlaga, M.L. Goldstein, F.B. McDonald, A.J. Lazarus, J. Geophys. Res. 90, 12027 (1985)

L.F. Burlaga, F.B. McDonald, N.F. Ness, A.J. Lazarus, J. Geophys. Res. 96, 3789 (1991)

L.F. Burlaga, F.B. McDonald, N.F. Ness, J. Geophys. Res. 98, 1 (1993)

H.V. Cane, G. Wibberenz, I.G. Richardson, T.T. von Rosenvinge, Geophys. Res. Lett. 26, 565 (1999)

E.W. Cliver, J. Geophys. Res. 98, 17435 (1993)

E.W. Cliver, A.G. Ling, Astrophys. J. Lett. 551, L189 (2001a)

E.W. Cliver, A.G. Ling, Astrophys. J. 556, 432 (2001b)

E.W. Cliver, A.G. Ling, Sol. Phys. (2010). doi:10.1007/s11207-010-9657-6

E.W. Cliver, O.C. St. Cyr, R.A. Howard, P.S. McIntosh, in Proc. 23rd Int. Cosmic Ray Conf., vol. 3 (1993), p. 517

E.W. Cliver, V. Boriakoff, K.H. Bounar, J. Geophys. Res. 101, 27091 (1996)

E.W. Cliver, A.G. Ling, I.G. Richardson, Astrophys. J. 592, 574 (2003)

N.U. Crooker, E.W. Cliver, J. Geophys. Res. 99, 23383 (1994)

J.A. Eddy, Science 192, 1189 (1976)

S.E.S. Ferreira, M.S. Potgieter, Adv. Space Res. 32, 657 (2003)

S.E.S. Ferreira, M.S. Potgieter, Astrophys. J. 603, 744 (2004)

S.E. Forbush, J. Geophys. Res. 59, 525 (1954)

S.E. Forbush, J. Geophys. Res. 63, 651 (1958)

L.J. Gleeson, W.I. Axford, Astrophys. J. Lett, 149, L115 (1967) 
N. Gopalswamy, S. Akiyama, S. Yashiro, P. Mäkelä, in Magnetic Coupling Between the Interior and the Atmosphere of the Sun, ed. by S.S. Hasan, R.J. Rutten. Astrophysics and Space Science Proceedings (Springer, Heidelberg, 2010), p. 289

B. Heber, Personal communication (2010)

B. Heber, M.S. Potgieter, Space Sci. Rev. 127, 117 (2006)

B. Heber, A. Kopp, J. Gieseler, R. Müller-Mellin, H. Fichtner, K. Scherer, M.S. Potgieter, S.E.S. Ferreira, Astrophys. J. 699, 1956 (2009)

A. Hewish, J. Geophys. Res. 106, 29409 (2001)

A.J. Hundhausen, J. Geophys. Res. 98, 13177 (1993)

A.J. Hundhausen, D.G. Sime, R.T. Hansen, S.F. Hansen, Science 207, 761 (1980)

J.R. Jokipii, D.A. Kopriva, Astrophys. J. 234, 384 (1979)

J.R. Jokipii, E.N. Parker, Astrophys. J. 160, 735 (1970)

J.R. Jokipii, B. Thomas, Astrophys. J. 243, 1115 (1981)

J.R. Jokipii, G. Wibberenz, Space Sci. Rev. 83, 365 (1998)

J.R. Jokipii, E.H. Levy, W.B. Hubbard, Astrophys. J. 213, 861 (1977)

J. Kóta, J.R. Jokipii, Astrophys. J. 265, 573 (1983)

A. Lara, N. Gopalswamy, R.A. Caballero-López, S. Yashiro, H. Xie, J.F. Valdéz-Galicia, Astrophys. J. 625, 441 (2005)

J.A. Le Roux, M.S. Potgieter, Astrophys. J. 361, 275 (1990)

J.A. Le Roux, M.S. Potgieter, Astrophys. J. 386, 336 (1992a)

J.A. Le Roux, M.S. Potgieter, Astrophys. J. 390, 661 (1992b)

J.A. Lockwood, J. Geophys. Res. 65, 19 (1960)

M. Lockwood, R. Stamper, M.N. Wild, Nature 399, 437 (1999)

M. Lockwood, A.P. Rouillard, I. Finch, R. Stamper, J. Geophys. Res. 111, A09109 (2006)

K.M. McCracken, J. Geophys. Res. 112, A09106 (2007)

F.B. McDonald, J.H. Trainor, N. Lal, M.A.I. Van Hollebeke, W.R. Webber, Astrophys. J. Lett. 249, L71 (1981)

F.B. McDonald, N. Lal, R.E. McGuire, J. Geophys. Res. 98, 1243 (1993)

P.S. McIntosh, in Astron. Soc. Pacific Conf. Series, ed. by K.L. Harvey. The Solar Cycle, vol. 27 (ASP, San Francisco, 1992), p. 14

H. Moraal, Space Sci. Rev. 19, 845 (1976)

H. Moraal, K.G. McCracken, Space Sci. Rev. (2011, this issue). doi:10.1007/s11214-011-9742-7

P. Morrison, Phys. Rev. 101, 1397 (1956)

K. Nagashima, I. Morishita, Planet. Space Sci. 28, 195 (1980)

E.N. Parker, Planet. Space Sci. 13, 9 (1965)

J.S. Perko, L.A. Fisk, J. Geophys. Res. 88, 9033 (1983)

M.S. Potgieter, Space Sci. Rev. 83, 147 (1998)

M.S. Potgieter, J. Atmos. Sol.-Terr. Phys. 70, 207 (2008)

M.S. Potgieter, Adv. Space Res. 46, 402 (2010)

M.S. Potgieter, J.A. Le Roux, Astrophys. J. 423, 817 (1994)

M.S. Potgieter, R.A. Burger, S.E.S. Ferreira, Space Sci. Rev. 97, 295 (2001)

I.G. Richardson, E.W. Cliver, H.V. Cane, J. Geophys. Res. 105, 18203 (2000)

I.G. Richardson, E.W. Cliver, H.V. Cane, Geophys. Res. Lett. 28, 2569 (2001)

I.G. Richardson, H.V. Cane, E.W. Cliver, J. Geophys. Res. 107, 1187 (2002), SSH 8-1

N.R. Sheeley Jr., T.N. Knudson, Y.-M. Wang, Astrophys. J. Lett. 546, L131 (2001)

E.J. Smith, J. Geophys. Res. 95, 18731 (1990)

E.J. Smith, B.T. Thomas, J. Geophys. Res. 91, 2933 (1986)

F. Steinhilber, J.A. Abreu, J. Beer, J.A. McCracken, J. Geophys. Res. 115, A01104 (2010)

L. Svalgaard, E.W. Cliver, J. Geophys. Res. 110, A12103 (2005)

L. Svalgaard, E.W. Cliver, J. Geophys. Res. 111, A09110 (2006)

L. Svalgaard, E.W. Cliver, Astrophys. J. Lett. 661, L203 (2007)

L. Svalgaard, E.W. Cliver, J. Geophys. Res. 115, A09111 (2010)

L. Svalgaard, Y. Kamide, E.W. Cliver, Geophys. Res. Lett. 32, L01104 (2005). doi:10.1029/2004GL021664 J.A. van Allen, Geophys. Res. Lett. 27, 2453 (2000)

Y.-M. Wang, N.R. Sheeley Jr., J. Geophys. Res. 99, 6597 (1994)

Y.-M. Wang, N.R. Sheeley Jr., A.P. Rouillard, Astrophys. J. 644, 638 (2006)

Y.-M. Wang, E. Robbrecht, N.R. Sheeley Jr., Astrophys. J. 707, 1372 (2009)

D.F. Webb, R.A. Howard, J. Geophys. Res. 99, 4201 (1994)

W.R. Webber, P.R. Higbie, J. Geophys. Res. 115, A05102 (2010a)

W.R. Webber, P.R. Higbie, J. Geophys. Res. (2010b). arXiv; 1003.4989 [physics.geo-ph]

W.R. Webber, P. Higbie, C.W. Webber, J. Geophys. Res. (2010). arXiv: 1004.2675 [physics.geo-ph]

G. Wibberenz, H.V. Cane, I.G. Richardson, T.T. von Rosenvinge, Space Sci. Rev. 97, 343 (2001)

G. Wibberenz, I.G. Richardson, H.V. Cane, J. Geophys. Res. 107, 1353 (2002) SSH 5-1 